\title{
A behavioral field approach to instrumental learning in the rat: I. Partial reinforcement effects and sex differences
}

\author{
PAUL T. P. WONG \\ Trent University, Peterborough, Ontario, K9J 7B8, Canada
}

\begin{abstract}
The behavioral field approach employs naturalistic observation and simultaneous, multiple recordings of ecologically relevant aspects of behavior-environment interactions. Applying this approach to runway learning in the rat, the inferior acquisition speed of partial reinforcement (PRF) subjects as compared to continuous reinforcement (CRF) subjects was found to result from greater response variability, more sniffing and goal-avoidance behavior, and slower dropping out of collateral behaviors (e.g., drinking and sand-digging). Extinction first produced an increase in exploratory behavior, then displacement activities (e.g., grooming and biting) and goal-avoidance. CRF subjects showed greater response persistence as measured by number of extinction trials to disrupt an established, favored path. PRF subjects showed greater goal persistence as measured by trials to retrace from goalbox. In extinction, while CRF subjects were more inclined to engage in drinking and sand-digging in the startbox, PRF subjects exhibited more biting behavior in the goalbox. The only sex-related differences were superior speeds by female CRF subjects, inferior goal speeds by female PRF subjects during acquisition, and superior goalbox escape learning by females in extinction.
\end{abstract}

The behavioral field approach to instrumental learning may be characterized by naturalistic observation and simultaneous multiple recordings of ecologically relevant aspects of behavior-environment interactions in a testing situation that permits the display of speciesspecific behavior; its focus of analysis is on the interrelationship between the designated instrumental response and other responses observed in the testing situation.

In contrast to the behavioral field approach, the traditional research strategy in animal learning calls for the arbitrary isolation of a small segment of environment (the stimulus) and a small segment of behavior (the response) and the analysis of functional relationship between them. To achieve this end, researchers typically employ a simple, straight runway or a barren operant chamber equipped with a protruding lever or a lighted key. In this simple-parts approach, highly complex behavioral phenomena are reduced to the analysis of some simple stimulus-response relationship quite arbitrarily chosen on the basis of convenience in manipulation and quantification. But focusing on simple parts

Supported by NRC Grant A8635 and a University Research Committee grant. The author gratefully acknowledges the assistance of Helen Orr in collecting the data, and the helpful comments of Robert Bolles, D. Bindra, Adam Carr, and Rod Wong on an earlier version of the paper. Requests for reprints should be addressed to Paul T. P. Wong, Department of Psychology, Trent University, Peterborough, Ontario, K9J 7B8, Canada. is often at the expense of observing a more complete picture of behavioral adaptation.

Consequently, the simple-parts approach has not been without critics. Bindra (1961) has marshalled a wide array of evidence to demonstrate the importance of investigating components of the "spontaneous" activity matrix from which the instrumental response stems. He argued that "the exact change in the performance of any given response brought about by a particular experimental manipulation (e.g., brain damage, stimulus change, drug injections) can be fully understood only if the effects of that manipulation on the occurrence of the components of general ability are known." Leeper (Note 1) proposed that learning might be a matter of elaboration and modification of existing larger complex units rather than simpler parts as hypothesized by Hull.

The conception of behavior as a unified, dynamic structure demands a different research strategy. Bindra and Joyce (1958) developed a time-sample method for measuring quantifiable categories of the components of general activity. Jensen (1970) suggested that "we must increase the amount and variety of information" by adopting the polythetic method, which involves the simultaneous observation and recording of several behavioral variables. In a similar vein, Bateson (1970) proposed that "rather than concentrate solely on the consequences of the animal's activities in its environment, such as 'rate of leverpressing' or 'arm of maze entered,' it is worth using other behavioral measures besides those traditionally used in studies of learning. Direct observation of the animals, providing 


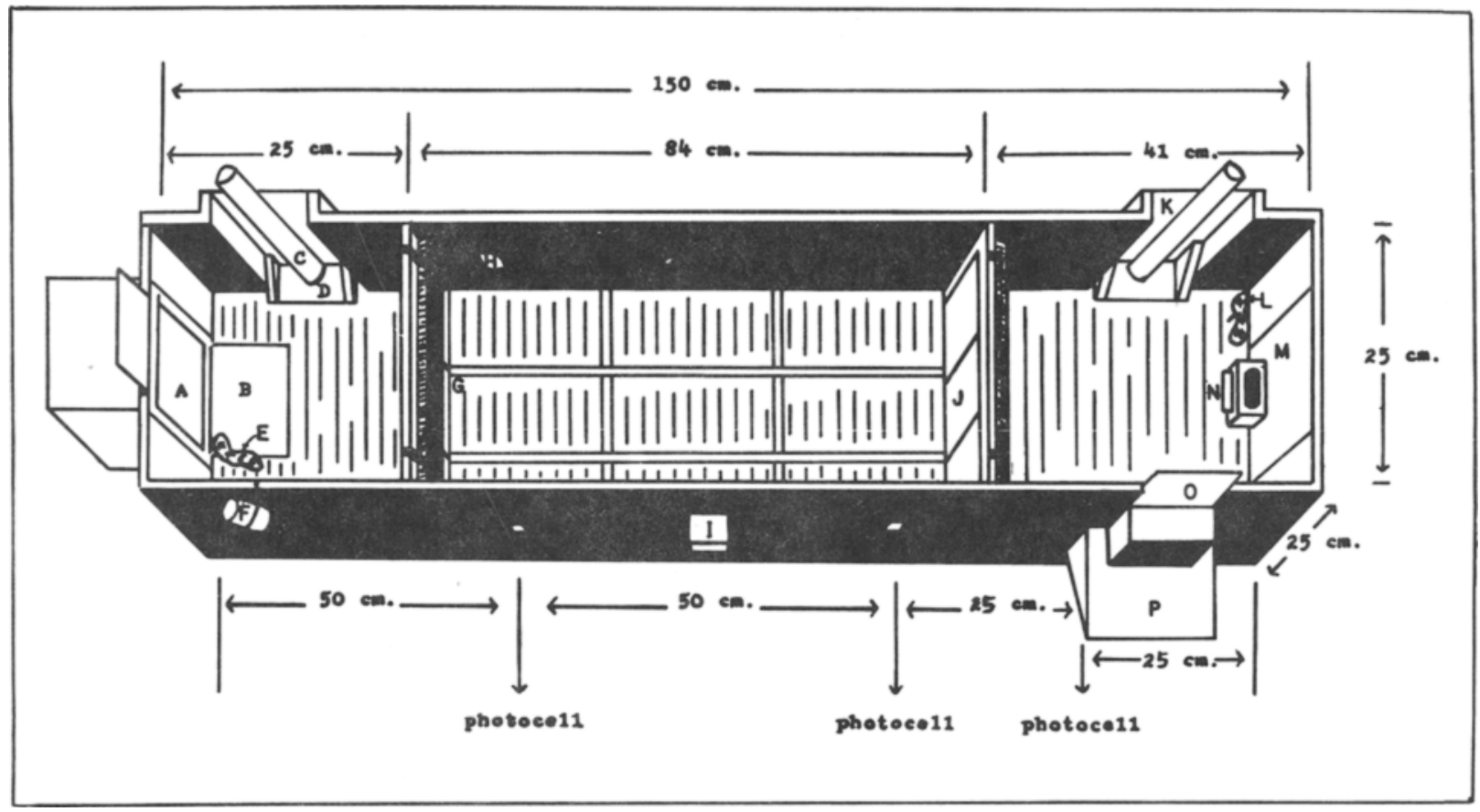

Figure 1. An overview of the basic version of the runway. (A) Entrance $(19 \times 8 \mathrm{~cm})$; (B) platform $(13 \times 14 \mathrm{~cm})$ resting on a microswitch $1 \mathrm{~cm}$ above the floor; (C) sandtube $(20 \mathrm{~cm}$ high); (D) base of the sand-digging apparatus $(5 \mathrm{~cm}$ wide, $6 \mathrm{~cm}$ deep, and $5 \mathrm{~cm}$ from the floor); (E) model animal; (F) drinking bottle; (G) rectangular box (4 $4.5 \times 25 \mathrm{~cm})$ containing three relay coil assemblies; (H) opening (6 x $6 \mathrm{~cm})$ leading to the exploring box; (I) exploring box $(9 \mathrm{~cm}$ wide, $9 \mathrm{~cm}$ high, $12 \mathrm{~cm}$ deep);(J) goaldoors $(15 \times 7.5 \mathrm{~cm}$ ); (K) sand-digging assembly; (L) model animal; (M) foodcup (5 cm long, $2 \mathrm{~cm}$ wide, $1.5 \mathrm{~cm}$ deep); (N) biting lids (5 cm long, $1 \mathrm{~cm}$ wide, $3 \mathrm{~cm}$ from floor); (0) escape door $(9.5 \times 23 \mathrm{~cm}) ;(P)$ metal canopy (24 x $23 \mathrm{~cm})$ with a boxlike metal enclosure $(9.5 \times 9 \times 11.5 \mathrm{~cm})$ mounted on it. Note-Features $H$ and $I$ were a later addition.

measures of the way it moves about, the kind of call it makes and so on, can yield important clues as to its state" (p. 563). Kantor (1970) criticized the traditional operant approach of reducing "all behavior to a single class adaptable to arbitrarily chosen patterns of manipulation and specialized apparatus," and proposed the analysis of behavioral fields.

Ethologists have long recognized that the criterion response in any situation is related to the particular behavioral context in which other responses also have certain probability of occurring (e.g., Andrew, 1956; Armstrong, 1950). Various techniques of simultaneous multiple recordings of behavioral events have also been developed (e.g., Bateson, 1968; Tobach, Schneirla, Aronson, \& Laupheimer, 1962).

While different in their orientations, these critics advocate an alternative approach which, with its common emphasis on observation and multiple recordings of various aspects of the behavioral context, may be called the behavioral field approach. In a limited way, this behavioral field approach has been applied to classical conditioning (Reynierse, Scavio, \& Ulness, 1970), operant conditioning (Dunham, 1971 ; Staddon \& Simmelhag, 1971), and discrete-trial instrumental learn. ing (Amsel, Wong, \& Scull, 1971). The present study constituted part of a larger research project designed to reexamine various established findings in discrete-trial instrumental learning through the analysis of behavioral fields.

\section{METHOD}

Subjects

The subjects were 18 male and 18 female albino rats ${ }^{1}$ of the Holtzman stock bred in the vivarium of Trent University, approximately 90 days old at the beginning of the experiment. They were housed in single cages with water always available and maintained on $70 \%$ of body weight adjusted for growth throughout the experiment.

\section{Apparatus}

The runway, with its inside dimensions, is presented in Figure 1. It was made of $1.2-\mathrm{cm}$-thick pressed board and covered with a Plexiglas lid. Two sets of swinging doors were made of metal sheets. Any of these doors may be kept open by a $12-\mathrm{V} \mathrm{dc}$ Guardian coil assembly (No. 20V-12D). Two sand-digging assemblies were installed in the runway. A detailed description of the sand-digging assembly can be found in Wong, Roach, and Osborne (1975). Two leather model seals, approximately $10 \mathrm{~cm}$ in length, obtainable from gift shops, were mounted on BRS omnidirectional levers fastened to the runway floor in an upright position. Lateral displacements of these levers, through biting the models, were recorded by digital counters. The drinking bottle in the startbox was connected with a drinkometer providing frequency of licking.

The depressing of the startbox platform started a clock, which, in conjunction with the activation of photobeams, provided three runway measures: start, run, and goal.

The lifting of the escape door disclosed an entrance $(4 \mathrm{~cm}$ 
above the runway floor, $8 \mathrm{~cm}$ wide, $19 \mathrm{~cm}$ high) leading to an escape cage $(23 \mathrm{~cm}$ wide, $24 \mathrm{~cm}$ long, $18 \mathrm{~cm}$ high) attached to the metal canopy. The lifting of the escape door started a clock, which was stopped by the closing of the escape door. providing the escape latency.

The entire runway floor was made of metal grids. A frame, made of $1.2-\mathrm{cm}$-thick, 3-cm-high pressed-board strips, was inserted only in the middle alley section, dividing it into nine equal segments. These segments were numerically coded to specify the exact route taken by each subject. A Sony videotape camera was mounted directly above the alley section to record the routes.

A manually operated four-key event recorder was connected to four cumulative timers to record the cumulative time subjects spent in each of the four mutually exclusive behavioral categories on any given trial.

\section{Procedure}

All subjects were reduced to and maintained at $70 \%$ of the average body weights of three ad-lib controls for each sex.

During runway habituation training, model animals and the drinking bottle were removed, the sand-digging units were blocked off by aluminum sheets, and rats of the same sex were always trained in pairs. The foodcup was always baited with 20 45-mg Noyes pellets on each trial; once one rat had consumed these pellets, the rat was removed and the foodcup was replenished with the same number of food pellets. On Day 1 of habituation training, all swinging doors were kept open, and each pair of rats was allowed a maximum of $5 \mathrm{~min}$ to freely explore the runway and consume the food pellets. On Day 2, rats that failed to consume food pellets were re-paired and given another session with a maximum of $60 \mathrm{~min}$. On Day 3 , the swinging doors were lifted only half way, about $6 \mathrm{~cm}$ from the floor. On Days 4 and 5, the swinging doors were closed and had to be pushed open by the rat. From Day 3 to Day 5 , a maximum of $5 \mathrm{~min}$ was allowed for each trial. Subjects that failed to push through the swinging doors by Day 5 were discarded.

During acquisition training, model animals were mounted, the drinking bottle was installed, and the sand-digging assemblies were made accessible. Subjects were given one trial per day for the first 2 days, and two trials per day thereafter.

To minimize contamination of sex odor, males were tested first as one squad, and $1 \mathrm{~h}$ later females were tested as another squad. For each squad, five random sequences were made up, and one of these sequences was randomly chosen as the running order for each testing day.

To start each trial, the subject was placed on the platform in the startbox, facing the center startdoor, and allowed a maximum of $3 \mathrm{~min}$ to enter the goalbox. Failing to traverse the runway in the allotted time, the subject was gently pushed into the goalbox, and a score of $60.00 \mathrm{sec}$ was accorded to any of the uncompleted runway measures. Guiding was typically used to help timid subjects push through the swinging doors during the initial stage of acquisition training.

Reward was in the form of four $45-\mathrm{mg}$ Noyes pellets. On rewarded $(R)$ trials, subjects were allowed to finish consuming the reward pellets and then given $30 \mathrm{sec}$ postreward goalbox confinement. On nonreward $(\mathrm{N})$ trials, subjects were confined in the unbaited goalbox for $30 \mathrm{sec}$. Thus, PRF and CRF subjects had the same opportunity to engage in collateral behavior in the goalbox.

PRF subjects were rewarded in $50 \%$ of the trials according to a predetermined quasi-random sequence with equiprobable $\mathrm{R}-\mathrm{R}, \mathrm{R}-\mathrm{N}, \mathrm{N}-\mathrm{R}, \mathrm{N}-\mathrm{N}$ transitions over eight blocks of four trials.

During extinction, the procedure was the same as during acquisition, except for the following: (a) If the subject failed to enter the goalbox in $3 \mathrm{~min}$, it was picked up and placed in the goalbox. (b) The subject was confined in the unbaited goalbox for $30 \mathrm{sec}$ before the escape door was lifted. (c) After the lifting of the escape door, which started the last timer, the subject was allowed a maximum of $120 \mathrm{sec}$ to escape from the goalbox. If a subject failed to escape within the allotted time, it was guided into the escape cage. Once the subject had hurdlejumped into the escape cage, the escape door was closed, and the last timer was automatically stopped.

On each triai, the experimenter started recording the time of engaging in each of the four categories of behavior as soon as the subject was placed in the startbox, and continued recording until the subject left or was removed from the goalbox. These four categories of behavior were:

(1) Activity -including any kind of instrumental activity that brings the animal into direct contact with various aspects of the testing environment. (This would include locomotive behavior, sand-digging, drinking, and biting.)

(2) Sniffing-head stretching and moving in the air or lowered towards the floor with whiskers twitching, with at least the hind part of the body stationary.

(3) Grooming-including any kind of self-directed activities, such as face-washing, licking, or scratching any part of the body.

(4) Immobility-crouching or sitting still with four paws on the floor and the body stationary except occasional slow and small lateral movements of the head.

These four broad categories of behavior can be objectively and easily identified, and interobserver agreement is very high (over 90\%).

\section{RESULTS AND DISCUSSION}

The focus will be first on the instrumental running response, and then on its interrelationship with other aspects of the behavioral field.

\section{Acquisition}

The instrumental response. Runway times are reciprocated and the speed data for both acquisition and extinction are shown in Figure 2. CRF groups exhibited a faster rate of acquisition as supported by a significant Reinforcement by Blocks interaction in start, run, and goal measures, Fs $=3.25,4.33,3.15$, respectively, $\mathrm{df}=7 / 744, \mathrm{p}<.01$ in all cases; the main effect of Reinforcement was significant only in the goal measure, $\mathrm{F}=6.12, \mathrm{df}=1 / 24, \mathrm{p}<.05$.

Females are faster in the start measure, resulting in a significant main effect of Sex, $F=6.85$, $\mathrm{df}=1 / 24$, $p<.05$. In the run and goal measures, females were faster only under CRF conditions as training progressed; and this was reflected by a significant Reinforcement by Sex by Blocks triple interaction, $F s=3.50,6.26$, respectively, $\mathrm{df}=7 / 744, \mathrm{p}<.01$ in both cases. This finding of female superiority under CRF conditions is consistent with the result of Beatty and O'Briant (1973). However, under PRF conditions, except for the start measure, there was little evidence of female superiority; in fact, female PRF subjects reached a lower asymptote than their male counterparts in the goal measure.

The overall pattern of sex differences during acquisition seems to suggest greater emotional reactivity to significant consequences in females: their faster approach under CRF conditions might be due to greater emotional reaction to reward; their slower goal approach under PRF conditions might be due to greater emotional 


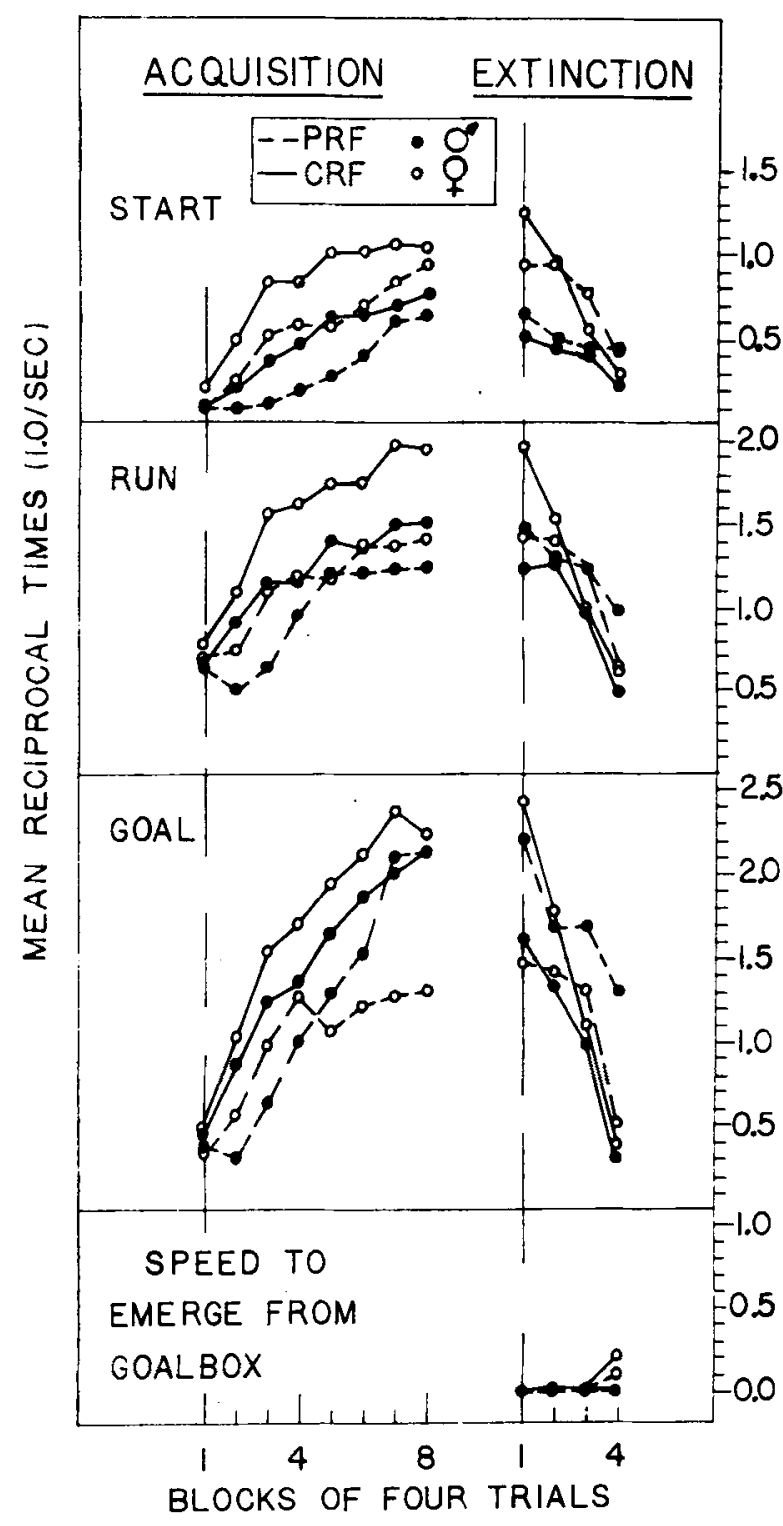

Figure 2. Speed data during acquisition and extinction.

reaction to nonreward. Research findings of lower aversive threshold to shock in female rats (Beatty \& Beatty, 1970; Paré, 1969) may also be considered as indicative of their greater emotional reactivity. However, in the open-field test, male rats seem to be more fearful as they defecate more and ambulate less (see Gray, 1971). Therefore, the issue of sex differences in emotional reactivity remains unresolved.

With respect to response routes, all subjects except one eventually fixed on a straight goalpath. PRF subjects showed greater variability in response patterns. Mean numbers of different routes for female PRF, CRF and male PRF, CRF groups were 10.28, 6.28, 9.57, and 5.14, respectively. Analysis of variance revealed a significant main effect of Reinforcement, $F=7.17$, $\mathrm{df}=1 / 24, p<.05$. This finding is consistent with the results of studies employing human subjects (Boroczi
\& Nakamura, 1964; McCray \& Harper, 1962; Newberry, 1971).

The subject was considered to be retracing only if it recoiled and turned $180^{\circ}$ away from the goalbox after traversing the entire alleyway and at least partially pushing open the swinging door. Mean frequencies of retracing for female PRF, CRF, and male PRF, CRF groups were $2.86,1.00,3.00$, and .45 , respectively. The main effect of Reinforcement was significant, $F=5.47$, $\mathrm{df}=1 / 24, \mathrm{p}<.05$.

According to Amsel (1958), frustration mediates response variation, avoidance, and other emotional behaviors. However, frustration theory (Amsel, 1962, 1967) has concentrated on the invigorating and inhibitory effects of frustration. Further research is needed to specify the exact conditions under which frustration produces avoidance or response variation. One of the relevant conditions might be proximity to the source of frustration or locus in the instrumental response chain. It is clearly adaptive to try a different route when a previous route has resulted in frustrative nonreward, and such a try-and-vary response tendency should be strongest at the very onset of the instrumental response chain. The avoidance tendency should be strongest close to the goalbox where conditioned frustration is more intense and the try-and-vary strategy is no longer relevant.

Analysis of the behavioral field. Proportions of time engaged in the four mutually exclusive categories of behavior that comprise the behavioral field are shown in Figure 3. Since grooming and freezing rarely occurred, analyses of variance were performed only on the activity and sniffing scores. It is worth noting that PRF, but not the $\mathrm{CRF}$, subjects exhibited a significant decrease in general instrumental activity $(F=6.91, d f=7 / 744$, $\mathrm{p}<.01)$ and a significant increase in sniffing $(\mathrm{F}=6.44$, df $=7 / 744, p<.01)$. The ecological significance of sniffing by PRF subjects was alluded to by Berlyne (1966, p. 25) when he observed that "an animal looking and sniffing around may stumble upon a clue to the whereabouts of food." According to Berlyne's analysis, lack of information or "irregularity" of stimulus events may provide the motivation for investigatory behavior. Partial reinforcement, being a case of uncertainty and irregularity in reinforcing events, should trigger a high level of sniffing, a species-typical investigatory behavior in rats.

Instrumental learning involves a process of narrowing the behavioral field through dropping out inefficient response patterns (i.e., long routes) and unproductive instrumental activities. This narrowing process was slower under PRF conditions, as evident in greater response variability and more collateral activities. ${ }^{2}$ Sand-digging data in acquisition are shown in the left side of Figure 4. PRF subjects spent more time sand-digging $(F=4.85, \mathrm{df}=1 / 24, \mathrm{p}<.05)$ and drinking in the startbox during acquisition. The mean frequencies 
of licking for PRF and CRF subjects were 10.14 and 1.50 , respectively $(U=26.5, p<.02) .^{3}$

Neither sand-digging nor drinking in the startbox can be considered as the schedule-induced adjunctive behavior which typically develops in the course of conditioning (Falk, 1971). In the present study, these collateral behaviors occurred mainly at the beginning of acquisition training in both CRF and PRF conditions, and decreased with training; perhaps they should be regarded as spontaneous species-typical behaviors of food-deprived rats in the testing situation. The slower dropping out of these collateral behaviors in PRF subjects may be due to slower growth of incentive or food expectancy.

In the goalbox, no systematic trend in sand-digging can be detected: while male groups showed a $\mathrm{U}$ function, female groups showed an increasing monotonic function, resulting in a significant Sex by Blocks interaction, $F=6.17, \mathrm{df}=7 / 168, \mathrm{p}<.01$. Previous studies have shown that sand-digging is positively

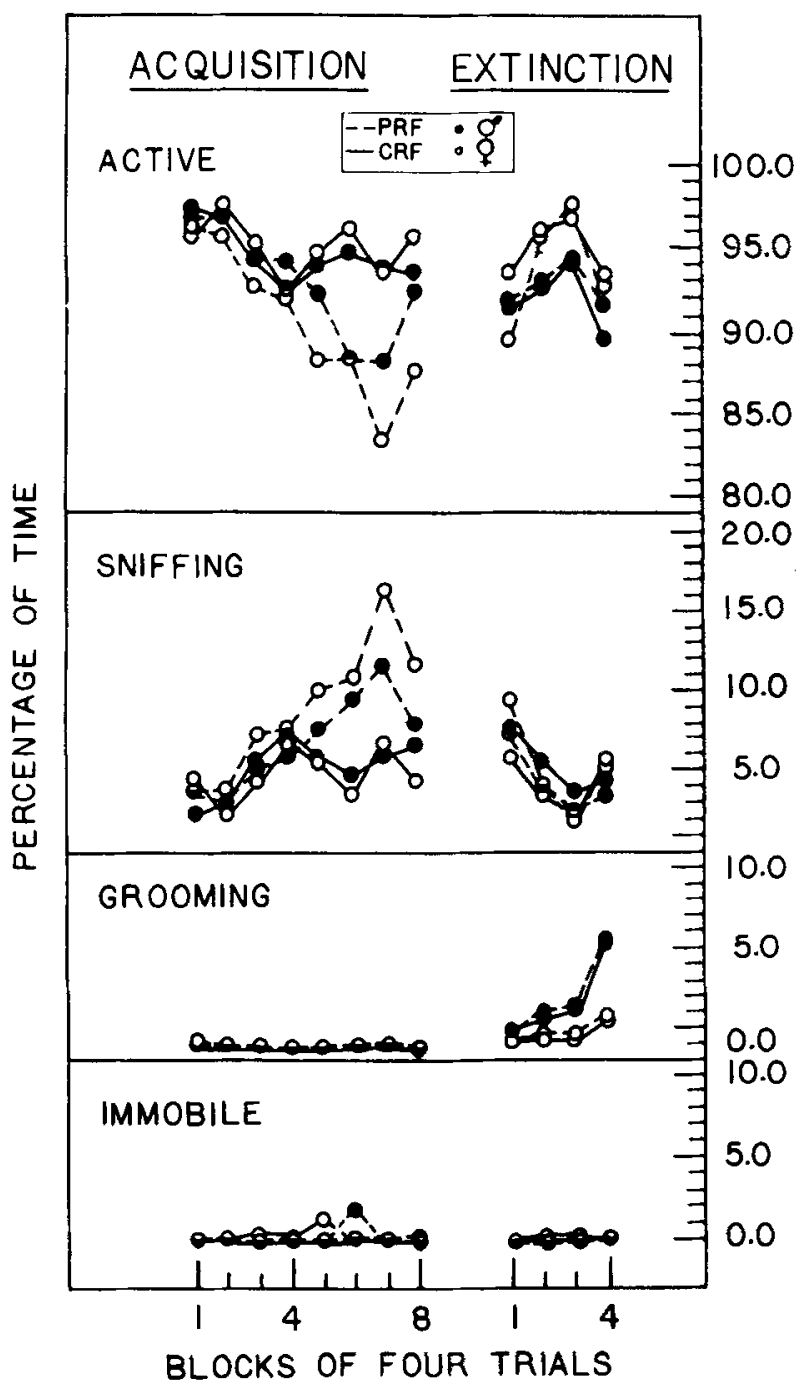
fields.

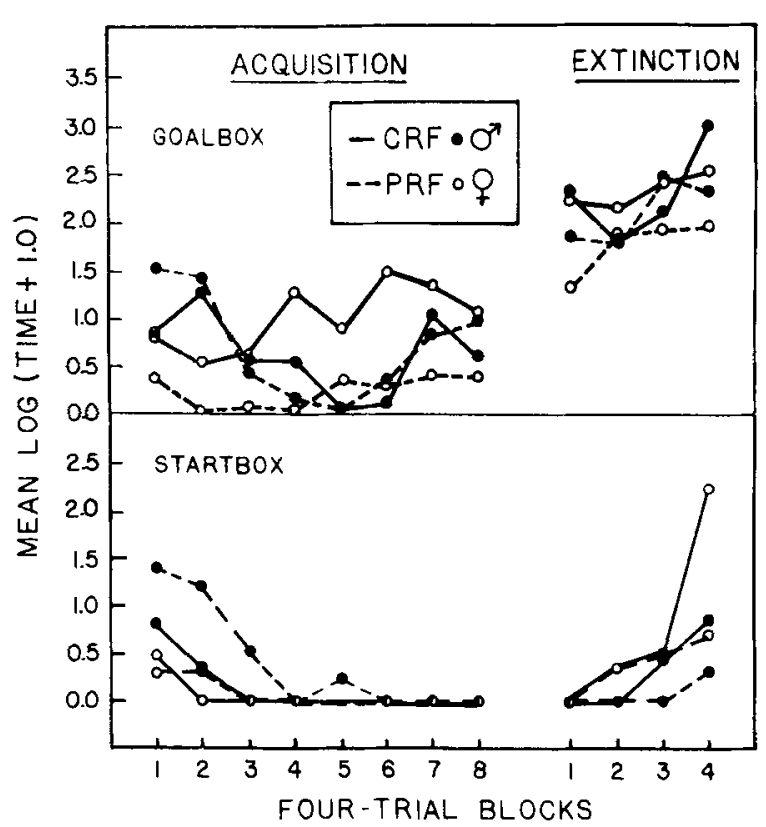
tion.

Figure 4. Sand-digging data during acquisition and extinc-

related to food deprivation (Earl, 1957; Fantino \& Cole, 1968; Wong, Roach, \& Osborne, 1975) and is rather insensitive to food-schedule manipulations in a free operant situation (White \& Wong, Note 2). In the present study, sand-digging most likely functioned as a hunger-generated species-specific food-gathering behavior, because subjects often picked up a grain of sand and chewed on it while digging.

The behavioral field approach has helped to identify four sources of interference that all contribute to the inferior acquisition performance of PRF subjects: greater response variability, more sniffing, more goalavoidance behavior, and slower dropping out of collateral behaviors.

In the present study, PRF groups did not overshoot their CRF counterparts in running speeds. However, it is predicted that once these four sources of competing responses are eliminated with extended acquisition training, a crossover would take place because of heightened drive attributable to anticipatory frustration (Amsel, 1967; Hug \& Amsel, 1969).

\section{Extinction}

The instrumental response. For both sexes, PRF groups eventually surpassed CRF groups during extinction in spite of CRF superiority during terminal acquisition (Figure 1). Analyses of variance revealed a significant Reinforcement by Blocks interaction in start, run, and goal measures, Fs $=5.00,7.77,9.21$, $\mathrm{df}=3 / 360, \mathrm{p}<.01$ in all three cases. However, the main effect of Reinforcement was not significant because of the carry-over of CRF superiority from acquisition to the initial stage of extinction.

The Sex main effect was significant only in the start measure $(F=5.69, \mathrm{df}=1 / 24, \mathrm{p}<.05)$. In the run and 
goal measures, sex differences existed only under CRF conditions, Fs $=5.86,4.25, \mathrm{df}=3 / 180, \mathrm{p}<.01$ in both cases. The fact that sex differences during extinction were obtained only in situations where these differences already existed during terminal acquisition clearly suggests a carry-over effect. This conclusion is indirectly supported by the finding that no sex differences were obtained in response persistence and goal persistence, both of which are not sensitive to differences in terminal acquisition speeds:

Response persistence. Specific response persistence or response perseveration refers to the number of extinction trials required to disrupt an established, favored path. Mean trials to response disruption ${ }^{4}$ for PRF and CRF groups were 6.25 and 10.71 , respectively, $F=9.44, \mathrm{df}=1 / 24, p<.01$. Response persistence was positively correlated with the frequency of occurrence of the favored path during acquisition (Pearson's $\mathrm{r}=$ +.55 ), and negatively correlated with response variability during acquisition $(\mathrm{r}=-.57)$.

The disruption of the favored path was followed by the use of alternative paths, demonstrating once again that frustrative nonreward generates response variation. Mean numbers of different responses for PRF and CRF subjects during extinction were 8.21 and 6.43 , respectively, and the difference was not statistically significant.

Goal persistence. In the present context, specific goal persistence refers to the pursuit of an initial goal by whatever route. It is measured by number of extinction trials to retrace from the goalbox. PRF subjects took significantly more trials ${ }^{5}$ to retrace than did the CRF subjects, 13.07 vs. $9.79, F=6.83, \mathrm{df}=1 / 24, \mathrm{p}<.05$. Retracing was not correlated with response variability during acquisition $(r=+.03)$ or the amount of reinforced practice of the favored route $(r=-.18)$.

The above two kinds of persistence may be independent. It is possible for a subject to exhibit high response persistence and low goal persistence; in this case, the subject persists in completing the favored path, but retraces from the goalbox after crossing the threshold and pushing open the goal door. In fact, five CRF subjects retraced before reaching the criterion of response disruption.

When trials to response disruption were subtracted from trials to retrace for each subject, ${ }^{6}$ it became clear that following response disruption, PRF subjects persisted longer in the try-and-vary response strategy before retracing $(\mathrm{F}=20.07, \mathrm{df}=1 / 24, \mathrm{p}<.01)$.

The present findings not only demonstrated the independence of these two kinds of persistence, but also showed that they were affected by different reinforcement histories in a different manner. While CRF training increased response persistence, PRF training increased goal persistence.

These two kinds of persistence are confounded in the speed measure, and cannot be disentangled in the traditional narrow, straight runway where "differences in the detailed movements or patterns of muscular activity from an occurrence to another are ignored, and all instances that produce the same environmental changes are treated as a single response class" (Spence, 1956, p. 42).

The present more molecular analysis of the extinction phenomenon suggests that response persistence is mainly determined by practice, while goal persistence may involve the conditioning of a try response strategy to cues of anticipatory frustration. In the presence of frustrative cues, especially at the onset of an instrumental response, the animal is assumed to experience the conflict of try or not try. Once the decision is made in favor of try, the animal may perform the same response more vigorously or switch to a different response. The acquisition finding of greater response variation by PRF subjects implicates the use of a try-and-vary response strategy. The conditioning of a try strategy to cues of anticipatory frustration should increase the tendency to enter the goalbox by different routes and decrease the tendency to avoid the goalbox (i.e., retrace), hence greater goal persistence in extinction. Since the try strategy is assumed to be a general coping behavior most relevant at the onset of instrumental response, the strategy-learning hypothesis predicts transsituational and transresponse transfer persistence, and the greatest amount of transfer should be obtained in the response initiation or start measure. This prediction has been supported by experiments not specifically designed to test the present strategy-learning hypothesis (McCuller, Wong, \& Amsel, 1976; Wong \& Amsel, 1976). This hypothesis also predicts greater persistence in employing the try-and-vary response strategy in extinction, and a weaker tendency to switch to collateral behaviors in the startbox. Results of the present experiment clearly support this prediction. The dropping out of retraces and the counterconditioning of goal approach to frustrative cues should also contribute to goal persistence in PRF subjects. But this counterconditioning hypothesis, stressing a more specific response topography (i.e., a locomotive approach response) does not predict transresponse transfer of persistence, nor does it predict the greatest amount of transfer in the latency of response initiation.

Goal escape. Speeds to hurdle-jump into the escape cage are shown in the last panel of Figure 1. Only female subjects learned to escape from the goalbox, resulting in a significant Sex by Blocks interaction, $\mathrm{F}=8.33$, $\mathrm{df}=$ $3 / 360, p<.01$. Several male subjects never left the goalbox within the allotted time of $2 \mathrm{~min}$ per trial. The present result corroborates previous findings of better avoidance learning by female rats (Beatty \& Beatty, 1970; Scouten \& Beatty, 1971).

Daly $(1969 a, b)$ demonstrated that rats learned a hurdle-jumping response to escape from cues associated with frustrative nonreward. Failure to learn the hurdleescape behavior by many subjects in the present study might be due to the opportunities to engage in sand-digging and biting in the goalbox.

Analysis of the behavioral field. Figure 3 reveals no 
reinforcement-related differences in all four aspects of behavioral fields. General instrumental activity first increased and then decreased, with females showing a slightly higher level of instrumental activity $(F=3.96$, $\mathrm{df}=1 / 24, p<.10)$. Sniffing decreased over trials ( $F=30.32, \quad \mathrm{df}=3 / 360, \quad \mathrm{p}<.01)$, while grooming increased $(\mathrm{F}=39.13, \mathrm{df}=3 / 360, \quad \mathrm{p}<.01)$, with males spending proportionally more time in grooming than females $(F=17.00, \mathrm{df}=1 / 24, \mathrm{p}<.05)$. The present finding of sex differences in general activity and grooming has been observed by others (see Gray, 1971, p. 84). It is also worth noting that grooming as a displacement activity (Tinbergen, 1952; Ziegler, 1964) rarely occurred during $P R F$ training which involves approach-avoidance conflict. It seems that grooming will become disinhibited (Andrew, 1956; Fentress, 1968) only when the conflicting approach and avoidance tendencies become equal in strength, a point likely to be reached in the course of extinction.

Closer examination of various components of the general instrumental activity indicated that its initial increase was mainly due to an increase in exploratory, locomotive behavior, because other collateral activities (e.g., drinking, biting) occurred mainly in the last extinction block, as shown in Figure 5. Evidence of increased response variability also supports this conclusion.

In the startbox, sand-digging increased over extinction trials $(F=7.86, \mathrm{df}=3 / 72, \mathrm{p}<.01)$, and so did drinking $(F=4.72, \quad d f=3 / 72, \quad p<.01)$. CRF subjects appeared more inclined to engage in drinking (mean frequency 15.07 vs. 3.50) and sand-digging (mean log time .56 vs. .24) than PRF subjects. However, these differences were not statistically significant, largely because subjects often engaged in only one of the two collateral activities, resulting in large variances. That CRF subjects spent more time in collateral activities in the startbox was aiso corroborated by the finding that four CRF subjects failed to leave the startbox within the maximum allotted time, while none of the PRF subjects failed. The observation of greater tendency to switch to collateral activities in the startbox by CRF subjects lends further support to the strategy-learning hypothesis that postulates an acquired stronger try-and-vary response tendency for PRF subjects.

In the goalbox, there were no significant sex-related or reinforcement-related differences in sand-digging in extinction. However, PRF subjects exhibited more model-biting than $\mathrm{CRF}$ subjects $(\mathrm{F}=7.38, \mathrm{df}=1 / 24$, $p<.05$ ). The fact that model-biting occurred only in extinction suggests that it might belong to the category of extinction-induced aggressive behavior (Azrin, Hutchinson, \& Hake, 1966; Thompson \& Bloom, 1966). It has been argued that if increased resistance to extinction following PRF training was due to increased frustration tolerance, PRF subjects should exhibit less extinction-induced aggression (Nevin, 1973). Why was there more biting behavior by PRF subjects in the

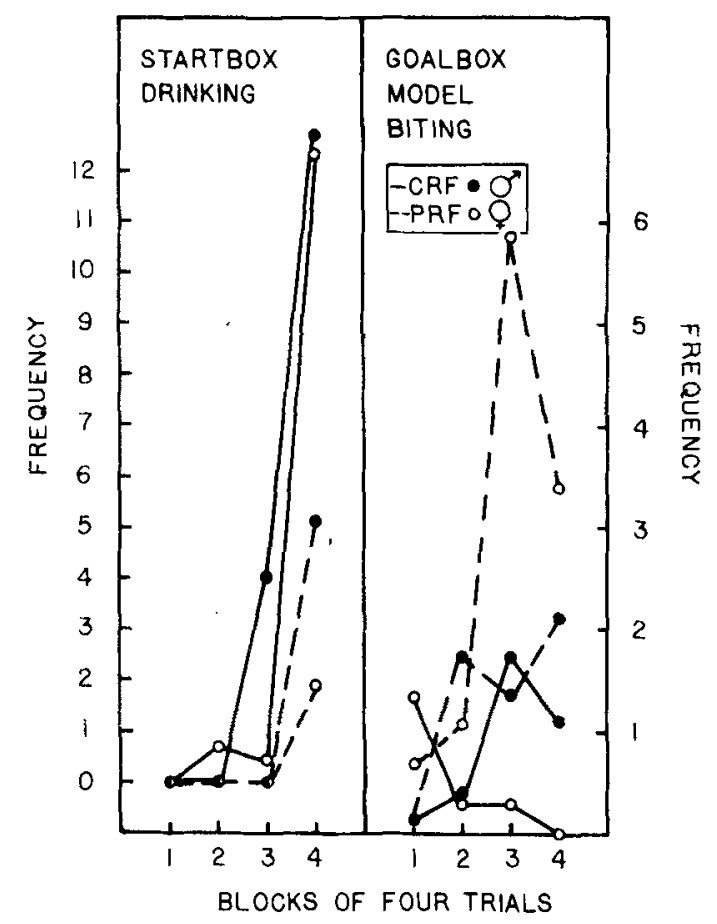

Figure 5. Drinking and model-biting data during extinction.

present study? One plausible explanation is that to approach and enter the goalbox on a subject's own accord involves more expectation of reward than being placed in the goalbox; therefore, nonreward following voluntary entry should be more frustrating and aggression-provoking. During the last two blocks of extinction, CRF subjects often failed to enter the goalbox within the allotted time, and had to be picked up and placed in the goalbox.

The present analysis of behavioral fields demonstrates the usefulness of observing and recording displacement activities in extinction (cf. Reynierse, Note 3). Manipulation of opportunities for collateral behaviors at different points of the designated instrumental response chain would shed further light on the extinction process.

\section{General Conclusion}

The present behavioral field approach to instrumental learning may lead to a more complete and ecologically more meaningful account of acquisition and extinction processes. The diversified information provided by the analysis of behavioral fields reduces the need for speculations about these processes. For example, at least some of the sources of interference responsible for inferior running speeds by PRF subjects are no longer a matter of theoretical speculation, but can be traced to various behaviors observed in the behavioral field.

Learning generally involves narrowing the behavioral field and the emergence of species-specific behavior relevant to the specific learning condition (e.g., sniffing and response variation by PRF subjects). Lorenz (1950) considered variable, appetitive instrumental behavior 
as one component of instinct. However, ethologists have been preoccupied with the hardcore of instinct, consum. matory behavior, while learning psychologists have favored the study of arbitrarily selected segments of instrumental behavior. The behavioral field approach, by combining naturalistic observation and experimentation, helps discover the instinctive components of instrumental behavior, thus bridging the gap between instinct and learning.

Extinction generally involves broadening the behavioral field but it involves more than the reemerging of behaviors that have dropped out in the course of acquisition. Displacement activities specific to the extinction-conflict situation (e.g., biting and grooming) also emerge. Further research on manipulation of timing, locale, and nature of displacement activities would provide more useful information on the extinction process.

The present approach has revealed two kinds of persistence. While specific response persistence seems to be related to the amount of practice during acquisition, goal persistence seems to stem from prior conditioning of a try-and-vary response strategy to anticipatory frustration cues. To identify factors that have differential effects on these two kinds of persistence would greatly increase our understanding of various extinction and transfer-of-persistence phenomena.

\section{REFERENCE NOTES}

1. Leeper, R. W. Hull and the myth of the simple-parts organism. Paper presented at the Annual Meeting of the American Psychological Association, September 1969, as part of the symposium on "The Hullian Impact on Psychology."

2. White, N., \& Wong, P. T. P. Schedule induced behavior under FT schedules of food or shock. Paper presented at the Annual Meeting of the Canadian Psychological Association, Quebec City, 1975.

3. Reynierse, J. H. A conflict and ethological theory of extinction. Paper presented at the Psychonomic Society Meetings, San Antonio, Texas, 1970.

\section{REFERENCES}

AMSEL, A. The role of frustration nonreward in noncontinuous reward situations. Psychological Bulletin, 1958, 55, 102-119.

AMSEL, A. Frustrative nonreward in partial reinforcement and discrimination learning: Some recent history and theoretical extension. Psychological Review, 1\%2, 69, 306-328.

AMSEL. A. Partial reinforcement effects on vigor and persistence: Advances in frustration theory derived from a variety of withinsubjects experiments. In K. W. Spence \& J. T. Spence (Eds.), The psychology of learning and motivation (Vol. I) Advances in research and theory. New York: Academic Press, 1967.

Amsel, A., Wong, P. T. P., \& SCull, J. Transfer of persistence in the domestic chick: Imprinting, punishment, and resistance to extinction of a food-rewarded running response. Psychonomic Science, 1971, 25, 174-176.

ANDREW, R. J. Some remarks on behavior in conflict situations, with special reference to Emberiza spp. British Journal of Animal Behaviour, 1956, 4, 41-45.

Armstrong, E. A. The nature and function of displacement activities. Symposium of the Society of Experimental Biology, $1950,4,361-386$.
Azrin, N. H., Hutchinson, R. R., \& Hake, D. F. Extinctioninduced aggression. Joumal of the Experimental Analysis of Behavior, 1966, 9, 191-204.

BAteson, P. P. G. Ethological methods of observing behavior. In L. Weiskrantz (Ed.), Analysis of behavioral change. New York: Harper \& Row, 1968. Pp. 389-399.

Bateson, P. P. G. Are they really the products of learning? In G. Horn \& R. A. Hinde (Eds.), Short-term changes in neural activity and behavior. Cambridge: Cambridge University Press, 1970.

Beatry, W. W., \& Beatty, P. A. Hormonal determinants of sex differences in avoidance and reactivity to electric shock in the rat. Journal of Comparative and Physiological Psychology, 1970, 73, 446-455.

Beatty, W. W., \& O'Briant, D. A. Sex differences in extinction of food-rewarded approach responses. Bulletin of the Psychonomic Society, 1973, 2, 97-98.

BERI YNE, D. E. Curiosity and exploration: Animals spend much of their time seeking stimuli whose significance raises problems for psychology. Science, 1966, 153, 25-33.

BINDRA, D. Components of general activity and the analysis of behavior. Psychological Review. 1961, 68, 205-225.

BindRA, D., \& Joyce, B. A time-sample method for measuring general activity and its components. Canadian Journal of Psychology, 1958, 12, 74-76.

Boroczi, G., \& NAKamURA, C. Y. Variability of responding as a measure of the effect of frustration. Journal of Abnormal and Social Psychology, 1964, 68, 342-345.

DALY, H. B. Learning of a hurdle-jump response to escape cues paired with reduced reward on frustrative nonreward. Journal of Experimental Psychology, 1969, 79, 146-157. (a)

DALY, H. B. Aversive properties of partial and varied reinforcement during runway acquisition. Joumal of Experimental Psychology, $1969,81,54-60$. (b)

Dunham, P. J. Punishment: Method and theory. Psychological Review, 1971, 78, 58-70.

EARL. R. W. Motivation, performance, and extinction. Journal of Comparative and Physiological Psychology, 1957, 50, 248-251.

FALK, J. L. The nature and determinants of adjunctive behavior. Physiology and Behavior, 1971, 6, 577-588.

Fantino, E., \& Cole, M. Sand-digging in mice: Functional autonomy? Psychonomic Science, 1968, 10. 29-30.

FENTRESS, J. C. Interrupted ongoing behavior in two speries of vole (Microtus agrestis and Clethrionomys britannicus): I. Response as a function of preceding activity and the context of an apparently "irrelevant" motor pattern. Animal Behaviour, 1968, 16. 135-153.

GRAY, J. A. The psychology of fear and stress. New York: McGrawHill, 1971.

HrLL, W. F. An attempted clarification of frustration theory. Psychological Review, 1968, 75, 173-176.

Hug, J. J., \& AMSEL, A. Frustration theory and partial reinforce. ment effects: The acquisition-extinction paradox. Psychological Review, 1969, 76, 419-421.

JễSẼN, D. D. Polythetic uiopsychology: An aiternative to behaviorism. In J. H. Reynierse (Ed.), Current issues in animal learning. Lincoln: University of Nebraska Press, 1970.

KANTOR, J. R. An analysis of the experimental analysis of behavior (TEAB). Joumal of the Experimental Analysis of Behavior, 1970. 13, 101-108.

LoRENz, K. The comparative method in the study of innate behavior patterns. Symposia of the Society for Experimental Biology, $1950,4,221 \cdot 268$.

MCCray, C. L., \& HaRper, R. S. Some relationships of schedules of reinforcement to variability of response. Joumal of Comparative and Physiological Psychology, 1962, 55, 19-21.

McCuller, T.. Wong, P. T. P..\& Amsel, A. Transfer of persistence from fixed-ratio barpressing to runway extinction. Animal Learning \& Behavior, 1976, 4, 53-57.

NEVIN. J. A. (Ed.) The study of behavior: Learning, motivation, emotion, and instinct. Glenview: Scott Foresman, 1973. 
NewberRy, B. H. Response variability and the partial reinforcement effect. Journal of Experimental Psychology, 1971, 89. 137-141.

PARÉ, W. P. Age, sex, and strain differences in the aversive threshold to grid shock in the rat. Journal of Comparative and Physiological Psychology, 1969, 69, 214-218.

Reynierse, J. H., Scavio, M. J., JR., \& Ulness, J. D. An ethological analysis of classically conditioned fear. In J. H. Reynierse (Ed.), Current issues in animal learning. Lincoln: University of Nebraska Press, 1970. Pp. 33-54.

Scouten, C. W., \& Beatry, W. W. Adrenocortical function and sex differences in acquisition and extinction of active avoidance behavior in the rat. Psychological Reports, 1971, 29, 1011-1018.

SPENCE, K. W. Behavior theory and conditioning. New Haven: Yale University Press, 1956.

Staddon, J. E. R., \& Simmelhag, V. L. The "superstition" experiment: A reexamination of its implication for the principles of adaptive behavior. Psychological Review, 1971, 78, 3.43.

Thompson, T., \& Bцоom, W. Aggressive behaviot and extinctioninduced response rate increase. Psychonomic Science, 1966, 5 , $335-336$.

Tinbergen, N. "Derived" activities. Their causation, biological significance, origin, and emancipation during evolution. Quarterly Review of Biology, 1952, 27, 1-32.

Tobach, E.. Schneirla, T. C., Aronson, C. R., \& LAUPheimer, R. The ATSL: An observer-to-computer system for a multivariate approach to behavioral study. Nature, 1962. 194, 257-258.

Wong, P. T. P., \& Amsel, A. Prior fixed ratio training and durable persistence in rats. Animal Learning \& Behavior, 1976 , 4. 461.466.

Wong. P. T. P., Ronch, T., \& Osborne, B. A sand-digging apparatus for rats. Behavior Research Methods \& Instrumentation, 1975, 7, 34-36.

ZeIGLER. H. P. Displacement activity and motivational theory: A case study in the history of ethology. Psychological Bulletin. 1964. 61. 362-376.

\section{NOTES}

1. Four males and four females were discarded because of sickness or failing to push through the swinging doors.

2 . In the present context, collateral behaviors refer to instrumental activities other than the criterion locomotive response. Since these collateral behaviors never occurred on every trial, their analyses were based on the sum of each collateral behavior in each block of four trials.

3. Because of the violation of homogeneity-of-variance assumption (Bartlett's test statistic $=32.46$ ), the Mann-Whitney $\mathrm{U}$ test was performed.

4. The path that was used more than $50 \%$ of the time in the last four blocks of acquisition trials was chosen as the favored path by the subject. For most subjects, the favored path was used almost consistently. The criterion for response-disruption was the disappearing of the favored path on two consecutive trials. This criterion was chosen because it allowed for occasional deviation from the favored path and because of the observation that once a favored path had disappeared on two consecutive trials, it no longer appeared with regularity.

5. Four PRF subjects never retraced. Therefore, the maximum number of extinction trials (16) was accorded to these subjects as trials to retrace.

6. When trials to retrace (goal persistence) were less than trials to response disruption (response persistence), as in the case of five CRF subjects, a negative value was obtained from such an abstraction operation. To eliminate negative numbers, a constant of 10 was added to each score. The resulting means for PRF and CRF groups were 16.86 and 9.43 , respectively.

(Received for publication March 29, 1976; revision received August 23, 1976.) 